\title{
The structure and evolution of scientific collaboration from the perspective of symbiosis
}

\author{
Junwan Liu ${ }^{1 *}$, Kaiyue Ding ${ }^{1}$, Feifei Wang ${ }^{1}$, Yi Bu² and Gregory J. Maus ${ }^{3}$ \\ ${ }^{1}$ School of Economics and Management, \\ Beijing University of Technology, Beijing, CHINA \\ ${ }^{2}$ Center for Complex Networks and Systems Research, School of Informatics, \\ Computing, and Engineering, Indiana University, Bloomington, IN., U.S.A. \\ ${ }^{3}$ School of Informatics, Computing, and Engineering, \\ Indiana University, Bloomington, IN., U.S.A. \\ e-mail: liujunwan@bjut.edu.cn* (corresponding author); \\ dingkaiyue@emails.bjut.edu.cn; feifeiwang@bjut.edu.cn; \\ uyi@iu.edu; gmaus@iu.edu
}

\begin{abstract}
This paper introduces a novel quantitative metric called the Symbiosis Degree Index for measuring the degree of symbiosis between subjects, which we apply it to analyzing scientific collaboration patterns between researchers. We study the network characteristics of scientific collaboration based on the resultant published research papers indexed in the Web of Science, considering the role of symbiosis. Analyses through our symbiosis metric of the relationship between a pair of mentor and mentee show substantively distinct ramifications from the "old leads young" model. In a sustained collaboration relationship, young researchers do indeed draw support from mentors' resources to improve their own level of scientific productivity and influence (as predicted in earlier models), but mentors also benefit by increasing their own productivity in publications. The collaborative symbiosis degree between mentor and mentee changes over time as the relationship matures. In the particular case studied here, even though the mentor's output decreased later in the relationship, their influence (measured through citations) continued to develop at the same pace through these collaborations. This is intended to demonstrate the potential of the metric to produce new insights on the nature of collaboration.
\end{abstract}

Keywords: Research collaboration; Scientific collaboration network; Symbiosis Degree Index; Mentormentee relationship; Collaborative productivity; Collaborative influence.

\section{INTRODUCTION}

Scientific research collaboration has always been an important force in scientific development: collaboration is conducive to the realization of knowledge complementarity, resource integration, and interdisciplinary research. However, scientific collaboration has transformed from the early point-to-point collaboration and chained collaboration pattern to the complex collaboration network mode (Milojević 2010), which resembles biological symbiosis between the collaborative subjects. This has prompted us to develop a theoretical framework and model to describe and explore the evolving networks. Currently, most research on the subject is focused on the network characteristics of scientific collaboration, 
and network theory has been used to study the structure and evolution of scientific collaboration (e.g., Bu et al. 2018a; Chen 2013; Newman 2001a; Zhang et al. 2018). However, network theory can only reveal the external characteristics of scientific collaboration and does not consider the intrinsic motivation for the collaboration itself. In contrast, symbiosis theory provides us with the appropriate perspective to consider such motivations. As discussed below, modern symbiosis theory is no longer confined to biology and has been extended to the fields of economics and social sciences, where it has been used to explain enterprise symbiosis relationships, industry-university-research symbiosis networks, etc.

\section{RELATED WORK}

\section{Analysis of Scientific Collaboration}

In the era of big science, scientific collaboration has become the main pattern of scientific research; thus, research on scientific collaboration has also emerged steadily. In the twentyfirst century, new research has been emerged in the field of scientific collaboration. In one of the highly cited foundational studies in the field (5016 citations in Google Scholar as of March 20, 2019), Newman (2001a) used the Medline database to investigate the structure of scientific collaboration networks in biological medicine, physics, and computer science. The results show that scientific collaborations exhibit high clustering properties, while the co-author networks present typical small-world phenomenon (Newman 2001a). Newman defined these collaboration networks in terms of publication co-authorship, a definition widely used in the field today. Research on structure of scientific collaboration using bibliometric indicators (such as the number of papers, average number of authors, number of collaborators, maximum size of the scientific collaboration team, and aggregation degree) published by Glänzel (2002) has received continued attention.

Since then, bibliometric indicators have been widely applied in the research of scientific collaboration networks (e.g., Bu et al. 2018b; Cavusoglu and Turker 2014; ChinchillaRodríguez et al. 2018; Wang et al. 2015). Social network analysis has also proven to be very effective for analyzing and visualizing co-authorship networks (e.g., Huerta-Barrientos et al. 2014; Otte and Rousseau 2002; White 2003; Kretschmer and Aguillo 2004). Subsequently, complex network analysis methods were used to investigate the structure of scientific collaboration networks, such as Ding (2011) which combined topic modeling and pathfinding algorithms to study the collaboration and citation tendencies of productive authors in the field of information retrieval. Social network techniques and bibliometric indicators have also been used in combination to analyze scientific collaboration (e.g., Hou et al. 2013; Zhang et al. 2018).

Research on the structure of scientific collaboration networks is a burgeoning subject in the field of scientometrics. Egghe (2003) and Newman (2001b) studied scientific weighted collaboration networks and indicated that the distribution of weights reflects the activity of academic exchanges in this field; specifically, they noted that active scientists tend to collaborate with or aid other active scientists. Qiu et al. (2014) utilized the frequency statistical method for the first time to construct a network of the collaborative age of scholars in the field of informatics.

Homophily and preferential attachment have also been observed as two common mechanisms in a network's evolutionary process. For these mechanisms, Wang and Zhu (2014) observed that as scientific collaboration networks evolve from early, middle, and late Page $\mid 60$ 
stages, their collaboration patterns correspondingly evolve from random selection to restrictive selection and finally to active selection. Zhang et al. (2018) examined the homophily effects of some bibliometric indicators (e.g., number of publications, number of citations, and gender) on forming new scientific collaboration relationships in the field of information retrieval.

\section{Symbiosis}

The concept of "symbiosis" was first proposed by German biologist Anton de Bary in 1879; this concept was associated with survival needs, where two or more organisms gradually form a mutually-beneficial relationship of coexistence and coevolution based on a pattern of interdependence and interaction (Douglas 1994). Yuan (1998) referenced the concept of symbiosis and constructed the framework of "symbiosis theory" in economic analysis, which he used to solve practical economic problems in China. In recent decades, the symbiosis theory has been gradually extended to the fields of Social Sciences and Management Science and has achieved initial success, resulting in some research hotspots such as "the industrial symbiosis network" (Wang et al. 2005; Albino et al. 2015; Domenech et al. 2011), "enterprise cluster symbiosis" (Wang et al. 2006), and "the industry-university-research symbiosis network" (Feng et al. 2013). Shi et al. (2013) applied the perspective of symbiosis theory, when conducting literature research, social network analysis, mathematical statistics, and quantitative analysis, to analyze the inter-provincial cooperation mechanism and the regional characteristics of sports scientific research in China. Smith (2011) performed a quantitative analysis of Wikipedia articles and their citations of documents in institutional repositories, revealing a potential symbiotic relationship between Wikipedia and academic research. However, there has still been very limited research for the measurement of the scientific cooperation based on the symbiotic theory, due to the lack of established quantitative metrics for degrees of symbiosis.

These previous findings on the structural characteristics and evolution trend of scientific collaboration networks obtained via social networking techniques are quite relevant to the scope of the present research. However, the symbiotic relationships within the framework of scientific collaboration have not been thoroughly investigated. One of the reasons is that the definition of symbiosis has not been agreed upon. Symbiosis is complex and has different types. Another reason is that mathematical models of symbiosis are often unrealistic or difficult to analyze (Williams 2013).

In scientific collaboration relationships, there is a complementarity between the partners regarding knowledge, expertise, and resources. By analyzing this mutual benefit to both participants as a form of symbiosis, researchers may gain insight into the motivations that drive collaboration. In this study, we reveal the scientific symbiosis network structure and its evolutionary trends using the symbiosis degree index by analyzing the scientific collaboration symbiotic relationship between academicians of the Chinese Academy of Sciences and their collaborators.

The Symbiosis Degree Index introduced here represents the first quantitative metric for distinguishing between different types and degrees of symbiotic relationships, at least as applied to analysis of collaborative research. 


\section{MATERIALS AND METHOD}

The academicians of the Chinese Academy of Sciences represent the highest academic level of Chinese scientists, playing an important role in the development of the country and its society, and enjoying high social prestige. In an earlier preliminary work, we studied the characteristics of a scientific collaboration network between academicians and found that there was a long-term and stable partnership between academicians and between academicians and non-academicians (Liu et al. 2015).

In this study, to examine the ongoing, collaborative mentor-mentee relationship, we looked for a typical example of such a pair who met the following criteria: (a) the two scientists collaborated for more than 15 years, and (b) they maintained close collaboration, that is, they published at least one co-authored paper a year.

The official website of the Chinese Academy of Sciences provides the basic information about the academicians such as gender, date of birth, place of origin, time of appointment, work units, as well as their main fields of research experience and awards. We cross-referenced this data with the ISI Web of Science database mapping it to their publications. We manually screened the search results according to their institutions, research areas, and other information regarding their published works to acquire the exact number of all their publications.

Of the pairs who met these criteria, we chose Li Shushen and Xia Jianbai to investigate the mentor-mentee collaboration pattern (shown in Table 1). They are academicians of the Information Technology and Science Department who have been working at the Institute of Semiconductors of the Chinese Academy of Sciences, maintaining a long-term relationship of frequent collaborations. Xia Jianbai obtained his master's degree from the Department of Physics at Peking University in 1965, and he was appointed as an academician of the Chinese Academy of Sciences in 2001. Li Shushen received his doctorate from the Institute of Semiconductors of the Chinese Academy of Sciences in 1996, and he was appointed as an academician of the Chinese Academy of Sciences in 2011. They published their first coauthored paper in 1994, when Li Shushen was a Ph.D. candidate at the Institute of Semiconductors, beginning their first collaboration as a mentor and a mentee. For the next 20 years, they maintained a collaborative relationship without interruption. Therefore, by investigating the co-authorship relation between $\mathrm{Li}$ and Xia, we are interested in addressing the following questions: Is there any significant change in the nature of the symbiotic relationship of scientific collaboration between a mentor and mentee in long-term collaboration? Moreover, we examined their relationship with our quantitative symbiotic metric.

Table 1. Characteristics of Symbiotic Subjects of the Mentor-Mentee Relationship

\begin{tabular}{cccccc}
\hline \hline Name & $\begin{array}{c}\text { Year of } \\
\text { birth }\end{array}$ & Title & $\begin{array}{c}\text { Time of being } \\
\text { appointed as an } \\
\text { academician }\end{array}$ & $\begin{array}{c}\text { Number of co- } \\
\text { authored papers }\end{array}$ & $\begin{array}{c}\text { Duration of } \\
\text { collaboration } \\
\text { (year) }\end{array}$ \\
\hline Xia Jianbai & 1939 & Academician & 2001 & 90 & 20 \\
Li Shushen & 1963 & Academician & 2011 & 90 \\
\hline \hline
\end{tabular}




\section{The Theoretical Lens}

Symbiosis is a self-organizing process, wherein mutual collaboration is established between the symbiotic units; moreover, there is complementarity and interdependence in terms of material resources, information, and energy. In biology, symbiosis is considered one of the fundamental mechanisms leading to species innovations that trigger species evolution. In sociology, the symbiotic relationship promotes economic innovation, technological innovation, and institutional innovation. From the viewpoint of scientific collaboration, a complementary relationship exists between knowledge, expertise, and resources among the collaborative subjects. The scientific collaboration relationship is symbiotic because there is continuous exchange of information and transmission of knowledge, which evolves with the collaboration. This produces new knowledge units, thereby transforming the collaboration structure.

Yuan (1998) explicitly put forward the theory of symbiosis which distinguishes the four types of symbiotic relationships generally accepted in biology parasitism (one subject benefits to the detriment of the other), commensalism (one subject benefits while another neither benefits nor suffers), symmetrical mutualism (both subjects receive the same benefit), and asymmetric mutualism (both subjects receive different benefits). Inspired by the definition of elasticity coefficient, which was introduced in the early 1970s and possibly earlier by Kacser and Burns (1973) in Edinburgh and Heinrich and Rapoport (1974) in Berlin, we quantify these different types of relationships by formulating the Symbiosis Degree Index (see details in Table 2), calculated through the following formula:

$$
R_{i}=\frac{\left(y_{i}-y_{i-1}\right) / y_{i-1}}{\left(x_{i}-x_{i-1}\right) / x_{i-1}}
$$

The symbiosis degree $R_{i}$ of symbiotic individuals in the period $i$ can be determined, where $x_{i}$ is the number of joint achievements of the symbiotic subject $A$ during the collaboration period $i$ (i.e., the number of collaborative papers published, the number of co-citation frequencies, etc.), and $y_{i}$ is the total number of achievements of the symbiotic subject $A$ in the scientific research during the period $i$ (total number of papers published, the number of citations, etc.). The formula reflects that the rate of change in all the achievements of a collaborator (total number of papers published or total citations) depends on the rate of change of the joint contributions (the number of co-authored papers or joint citations) that are obtained through collaboration.

While this formula has applications in many fields, in this paper we will apply it to analyzing the roles of scientific collaboration in terms of the academic output (number of papers) or influence (citation frequency) of an academician. The symbiosis degree contributing to the scientific collaboration indicator reflects the extent of the benefit of the collaboration to the scientific research symbiotic subjects as well as the extent of the dependence of the symbiotic subjects on the collaborative relationship. Here, the number of individual publications and the citation frequency represent an individual's research productivity and influence respectively. When $x_{i}$ represents the number of papers, $R_{i}$ represents the degree to which the symbiotic relationship contributes to scientific productivity. When $x_{i}$ represents the citation frequency, $R_{i}$ represents the degree to which the symbiotic relationship contributes to scientific influence.

The extent of dependence of the two symbiotic subjects ( $A$ and $B$ ) on the scientific collaboration in different periods is calculated using the symbiosis degree index $(R(A)$ and 
$R(B)$, respectively).

In practice it is unlikely that $\mathrm{R}(\mathrm{A})=\mathrm{R}(\mathrm{B})$, so moreover, when $0.95<R(A) / R(B) \mid<1.05$, we consider that the values of $R(A)$ and $R(B)$ to be sufficiently close that we consider it a symmetrical mutualism relationship, only if both $R(A)$ and $R(B)$ is great than 0 . When either $R(A)$ or $R(B)$ less than 0 , it is a parasitic relationship between the two rather than a symmetrical mutualism relationship.

Table 2 presents the four types of symbiotic relationship established between $A$ and $B$ under different conditions.

Table 2. Symbiosis Degree Index: Quantifying the Symbiotic Relationship in Terms of Behavior.

\begin{tabular}{|c|c|c|}
\hline Numerical comparison & $\begin{array}{l}\text { Symbiotic } \\
\text { relationship }\end{array}$ & Characteristics \\
\hline$R(A)>0, R(B)>0$ & Mutualism & $\begin{array}{l}\text { When the symbiosis degree index is positive and the } \\
\text { values are not equal, the two symbiotic subjects have } \\
\text { an asymmetrical mutualism relationship; if the values } \\
\text { are near-equal, then they have an asymmetrical } \\
\text { mutualism symbiotic relationship }\end{array}$ \\
\hline $\begin{array}{l}R(A)=0, R(B)>0 \text { or } \\
R(A)>0, R(B)=0\end{array}$ & Commensalism & $\begin{array}{l}\text { One of the two symbiotic subjects benefits from the } \\
\text { collaborative symbiotic relationship, while the other } \\
\text { does not }\end{array}$ \\
\hline $\begin{array}{l}R(A)<0, R(B)>0, \text { or } \\
R(A)>0, R(B)<0\end{array}$ & Parasitism & $\begin{array}{l}\text { One subject benefits to the detriment of the other } \\
\text { subject }\end{array}$ \\
\hline $\begin{array}{l}R(A)<0, R(B)<0 \text { or } \\
R(A)=0, R(B)<0 \text { or } \\
R(A)<0, R(B)=0 \text { or } \\
R(A)=R(B)=0\end{array}$ & $\begin{array}{l}\text { No symbiotic } \\
\text { relationship }\end{array}$ & $\begin{array}{l}\text { The two symbiotic subjects do not depend on the } \\
\text { collaboration, the collaboration is not beneficial to the } \\
\text { symbiotic subjects, and a real collaborative symbiotic } \\
\text { relationship almost does not exist }\end{array}$ \\
\hline
\end{tabular}

\section{RESULTS AND DISCUSSION}

\section{Evolutionary Characteristics of the Collaborative Symbiotic Network between a Mentor and Mentee}

Based on the collected data, we use the Derwent Data Analyzer (DDA) (Clarivate Analytics 2017) to clean the data, extract the author's information regarding their publications, and then create the collaboration matrix between the academicians after further processing. Derwent Data Analyzer (DDA) is an intelligent information analysis tool providing multi-angle data mining and visualization of panoramic analysis. It can transform literature or patent search data into a fully integrated data set for analysis. In this paper, we use DDA's data transforming function to gain the co-occurrence matrix between authors, which is the basis of collaboration network drawing. Because of the huge size of data, only some of the author's co-occurrence matrix is intercepted for scholars' reference, part of the co-occurrence matrix is shown in Table 3.

Ucinet (Borgatti, 2002) is used for the network index analysis and for drawing the coauthorship network map at each time point. Figure 1 shows the collaboration network between academicians Xia Jianbai and Li Shushen. In this figure, we observe that the two academicians had their own collaboration networks in different periods and maintained a Page | 64 
strong collaborative relationship. In Figure 1, the reason why such a division of the time period between mentor and mentee, based on before and after mentor and mentee elected as academicians, taking every five years for a stage. The mentor Xia Jianbai was appointed as an academician in 2001, the mentee Li Shushen was appointed as an academician in 2011, so we take 2001 and 2011 as the time point to explore the mentor-mentee symbiotic relationship over time.

Table 3. Partial Collaboration Matrix (the number in the matrix shows the number of collaborations between two given authors)

\begin{tabular}{lcccccccccc}
\hline \hline & Xia, & Li, & Li, & Chang, & Zhang, & Wei, & Niu, & Wang, & Jiang, & Fu, \\
& JB & SS & JB & $\mathbf{K}$ & $\mathbf{P}$ & $\mathbf{S}$ & $\mathbf{Z}$ & $\mathbf{L}$ & $\mathbf{X}$ & $\mathbf{Z}$ \\
\hline \hline Xia Jianbai & 223 & 86 & 35 & 19 & 1 & 14 & 8 & 8 & 2 & 0 \\
Li Shushen & 86 & 216 & 43 & 4 & 18 & 16 & 11 & 15 & 11 & 10 \\
Li Jingbo & 35 & 43 & 47 & 0 & 0 & 16 & 0 & 5 & 2 & 0 \\
Chang Kai & 19 & 4 & 0 & 19 & 0 & 0 & 0 & 0 & 0 & 0 \\
Zhang Ping & 1 & 18 & 0 & 0 & 18 & 0 & 0 & 0 & 0 & 9 \\
Wei Suhuai & 14 & 16 & 16 & 0 & 0 & 17 & 0 & 1 & 0 & 0 \\
Niu Zhichuan & 8 & 11 & 0 & 0 & 0 & 0 & 16 & 0 & 0 & 0 \\
Wang Linwang & 8 & 15 & 5 & 0 & 0 & 1 & 0 & 15 & 8 & 0 \\
Jiang Xiangwei & 2 & 11 & 2 & 0 & 0 & 0 & 0 & 8 & 11 & 0 \\
Fu Zhenguo & 0 & 10 & 0 & 0 & 9 & 0 & 0 & 0 & 0 & 10 \\
\hline \hline
\end{tabular}

Note: The first rows present the abbreviated names of the authors' full names in the first columns in the matrix. The abbreviated names are also used in the following figures and visualizations.

After conducting centrality analysis of the collaborative relationship between the two academicians, the centrality of the network is obtained by ranking the top five co-authors, as shown in Table 4. After analyzing the data, we can conclude that in the collaboration network, the centrality degree of Li Shushen is higher than that of Xia Jianbai and that the closeness centrality and betweenness centrality of Xia Jianbai are higher than Li Shushen's. At the same time, another researcher Li Jingbo maintained a close collaboration with both academicians.

Table 4. Centrality Analysis of the Two Academicians' Scientific Collaboration Network.

\begin{tabular}{lrlrlr}
\hline \hline \multicolumn{1}{c}{ The top five } & \multicolumn{1}{c}{$\begin{array}{c}\text { Degree } \\
\text { centrality }\end{array}$} & The top five & $\begin{array}{r}\text { Closeness } \\
\text { centrality }\end{array}$ & The top five & $\begin{array}{r}\text { Betweenness } \\
\text { centrality }\end{array}$ \\
\hline \hline Li Shushen & 2.064 & Xia Jianbai & 73.251 & Xia Jianbai & 62.306 \\
Xia Jianbai & 1.983 & Li Shushen & 69.531 & Li Shushen & 51.037 \\
Li Jingbo & 0.657 & Li Jingbo & 53.776 & Li Jingbo & 1.643 \\
Niu Zhichuan & 0.271 & Niu Zhichuan & 52.353 & Zhang Ping & 1.574 \\
Wei Suhuai & 0.261 & Wei Suhuai & 52.047 & Chang Kai & 1.358 \\
\hline \hline
\end{tabular}

Table 4 analyzes the network analysis results of top five scholars. The analysis results in this table are based on the network analysis results of all Science Citation Index (SCl) papers published by Li and Xia. Li has published 207 papers and Xia has published 197 papers, so the data analysis is based on the papers published by the two academicians rather than the papers published in the field. Thus, the top two ranking in the network are Li and Xia, which is reasonable. The specific collection process is to search the Web of science database with the full name and abbreviation of the English names of the two academicians of Li and Xia, and to refine the search results by using the academic institution (including the former 
institutions) and the research field, in this way, delete the wrongly matched papers brought by the academician's name.

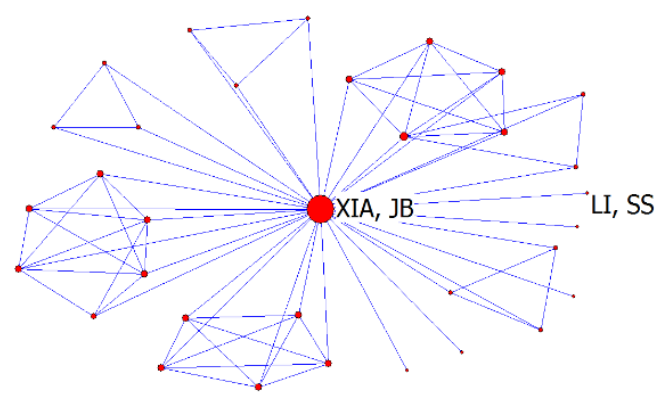

(a) 1994-1995

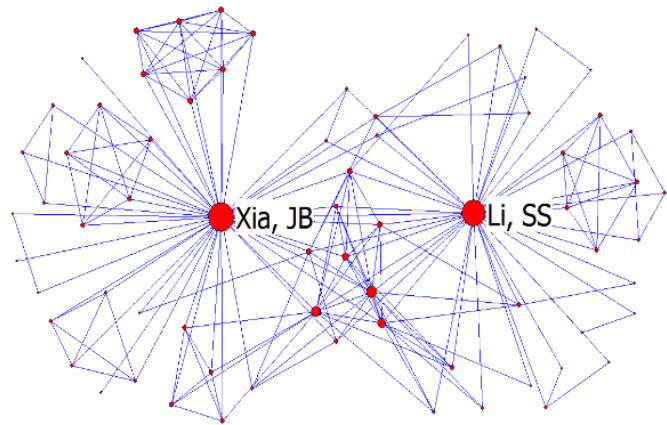

c) 2001-2005

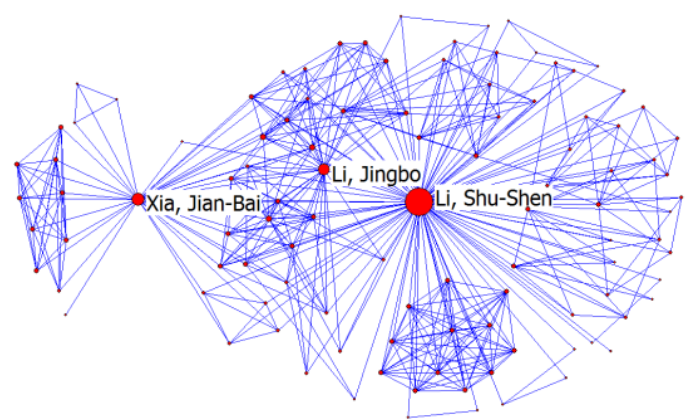

(e) 2011-2014

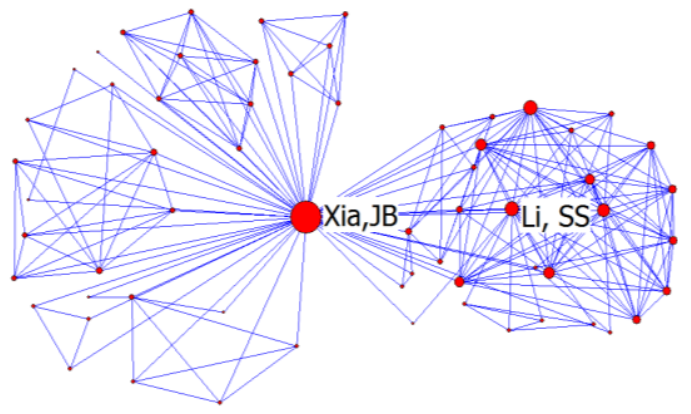

(b) $1996-2000$

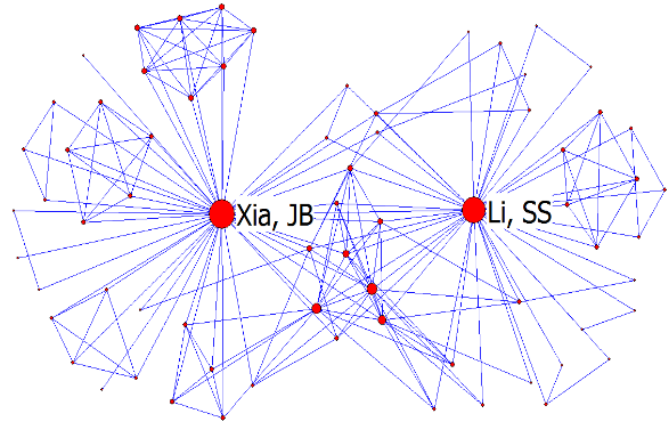

(d) $2006-2010$

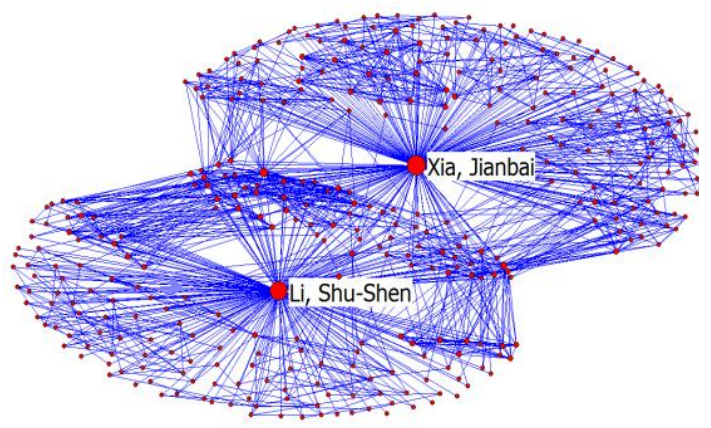

(f) Overall collaboration network

Figure 1. Scientific Collaboration Network between Xia Jianbai (Xia JB) and Li Shushen (Li SS).

\section{Collaborative Symbiotic Relationship between a Mentor and a Mentee}

To explore the evolutionary process of the symbiotic relationship between the two academicians over the course of 20 years, we compare the scientific collaboration symbiosis degree indices (number of co-authored papers and joint citation frequency). Based on the time-series distribution of their number of collaborative papers and the joint citation frequency of the two academicians for the last 20 years (Table 5). Both indices gradually increased, reaching a peak in 2006-2010; then, they decreased. 
Table 5. Distribution of the Number and the Citation Frequency of Co-authored Papers Published by the Two Academicians.

\begin{tabular}{lccccc}
\hline \hline \multicolumn{1}{c}{ Period } & $\mathbf{1 9 9 4 -}$ & $\mathbf{1 9 9 6 -}$ & $\mathbf{2 0 0 1 -}$ & $\mathbf{2 0 0 6 -}$ & $\mathbf{2 0 1 1 -}$ \\
& $\mathbf{1 9 9 5}$ & $\mathbf{2 0 0 0}$ & $\mathbf{2 0 0 5}$ & $\mathbf{2 0 1 0}$ & $\mathbf{2 0 1 4}$ \\
\hline \hline Number of co-authored papers & 2 & 8 & 16 & 47 & 17 \\
Total number of papers of Li Shushen & 2 & 12 & 48 & 80 & 65 \\
Total number of papers of Xia Jianbai & 7 & 47 & 40 & 78 & 25 \\
Joint citations & 30 & 322 & 505 & 1408 & 166 \\
Total citations of Li Shushen & 30 & 354 & 856 & 1759 & 174 \\
Total citations of Xia Jianbai & 86 & 979 & 829 & 1669 & 172 \\
\hline \hline
\end{tabular}

Based on the above statistical results, the number of papers represents the scientific research productivity of the academician, and the citation frequency represents the scientific research influence. We calculate the symbiosis degree of scientific collaboration productivity and influence of the collaborative papers of the two academicians in five periods between 1994 and 2014 (Tables 6 and 7, respectively). In these two tables, R(A) represents the symbiosis degree of the scientific collaboration of Li Shushen, and $R(B)$ represents the symbiosis degree of the scientific collaboration of Xia Jianbai. The productivity symbiosis degree formula needs to calculate the difference between the number of papers in the current period and that in the previous period. Thus, the calculation of the symbiosis degree in the first period cannot use the formula, so when calculating the symbiosis degree of the first period, we adopt the ratio of the number of collaborative papers to the total number of papers of the symbiotic unit in the first period as the calculation method of the symbiosis degree. To a certain extent, it also conforms to the connotation of symbiosis degree, that is, to measure the degree of dependence of symbiotic units on collaboration in a certain period. The same applies to influence symbiosis degree.

Table 6. Symbiosis Degree of Collaborative Productivity between Mentor and Mentee.

\begin{tabular}{|c|c|c|c|c|c|}
\hline Period & $\begin{array}{c}\text { Li } \\
\text { Shushen } \\
{[R(A)]}\end{array}$ & $\begin{array}{c}\text { Xia } \\
\text { Jianbai } \\
{[R(B)]}\end{array}$ & $\begin{array}{l}\text { Numerical } \\
\text { comparison }\end{array}$ & $\begin{array}{l}\text { Judgment of } \\
\text { symbiotic } \\
\text { relationship }\end{array}$ & Characteristics \\
\hline $\begin{array}{l}\{1994, \\
1995\}\end{array}$ & 1 & 0.29 & $R(A)>R(B)$ & $\begin{array}{l}\text { asymmetric } \\
\text { mutualism }\end{array}$ & $\begin{array}{l}\text { Li Shushen's dependence on } \\
\text { collaboration is greater than } \\
\text { that of Xia Jianbai }\end{array}$ \\
\hline $\begin{array}{l}\{1996 \\
2000\}\end{array}$ & 1.67 & 1.9 & $R(A)<R(B)$ & $\begin{array}{l}\text { asymmetric } \\
\text { mutualism }\end{array}$ & $\begin{array}{l}\text { Xia Jianbai's dependence on } \\
\text { collaboration is slightly } \\
\text { greater than that of Li } \\
\text { Shushen }\end{array}$ \\
\hline $\begin{array}{l}\{2001 \\
2005\}\end{array}$ & 3 & -0.15 & $R(A)>R(B)$ & commensalism & $\begin{array}{l}\text { Li Shushen's dependence on } \\
\text { collaboration is much greater } \\
\text { than that of Xia Jianbai }\end{array}$ \\
\hline $\begin{array}{l}\{2006 \\
2010\}\end{array}$ & 0.34 & 0.49 & $R(A)<R(B)$ & $\begin{array}{l}\text { asymmetric } \\
\text { mutualism }\end{array}$ & $\begin{array}{l}\text { Xia Jianbai's dependence on } \\
\text { collaboration is slightly } \\
\text { greater than that of } \mathrm{Li} \\
\text { Shushen }\end{array}$ \\
\hline $\begin{array}{l}\{2011, \\
2014\}\end{array}$ & 0.29 & 1.06 & $R(A)<R(B)$ & $\begin{array}{l}\text { asymmetric } \\
\text { mutualism }\end{array}$ & $\begin{array}{l}\text { Xia Jianbai's dependence on } \\
\text { collaboration is greater than } \\
\text { that of Li Shushen }\end{array}$ \\
\hline
\end{tabular}


Table 7. Symbiosis Degree of Collaborative Influence between Mentor and Mentee.

\begin{tabular}{|c|c|c|c|c|c|}
\hline Period & $\begin{array}{c}\text { Li } \\
\text { Shushen } \\
{[R(A)]} \\
\end{array}$ & $\begin{array}{c}\text { Xia } \\
\text { Jianbai } \\
{[R(B)]}\end{array}$ & $\begin{array}{l}\text { Numerical } \\
\text { comparison }\end{array}$ & $\begin{array}{l}\text { Judgment of } \\
\text { symbiotic } \\
\text { relationship }\end{array}$ & Characteristics \\
\hline $\begin{array}{l}\{1994, \\
1995\}\end{array}$ & 1 & 0.35 & $R(A)>R(B)$ & $\begin{array}{l}\text { asymmetric } \\
\text { mutualism }\end{array}$ & $\begin{array}{l}\text { Li Shushen's } \\
\text { dependence on the } \\
\text { collaboration is } \\
\text { greater than that of } \\
\text { Xia Jianbai }\end{array}$ \\
\hline $\begin{array}{l}\{1996 \\
2000\}\end{array}$ & 1.009 & 1.006 & $\begin{array}{c}\mathrm{R}(\mathrm{A})>\mathrm{R}(\mathrm{B}) \\
0.95<|R(A) / R(B)|<1.05\end{array}$ & $\begin{array}{l}\text { symmetric } \\
\text { mutualism }\end{array}$ & $\begin{array}{l}\text { The difference in the } \\
\text { dependence on the } \\
\text { collaboration } \\
\text { between the two is } \\
\text { very small }\end{array}$ \\
\hline $\begin{array}{l}\{2001 \\
2005\}\end{array}$ & 1.6 & -0.5 & $R(A)>R(B)$ & commensalism & $\begin{array}{l}\text { Li Shushen's } \\
\text { dependence on the } \\
\text { collaboration is much } \\
\text { greater than that of } \\
\text { Xia Jianbai }\end{array}$ \\
\hline $\begin{array}{l}\{2006, \\
2010\}\end{array}$ & 0.8 & 0.78 & $\begin{array}{c}\mathrm{R}(\mathrm{A})>\mathrm{R}(\mathrm{B}) \\
0.95 \triangleleft R(A) / R(B) \mid<1.05\end{array}$ & $\begin{array}{l}\text { symmetric } \\
\text { mutualism }\end{array}$ & $\begin{array}{l}\text { The difference in the } \\
\text { dependence on the } \\
\text { collaboration } \\
\text { between the two is } \\
\text { very small }\end{array}$ \\
\hline $\begin{array}{l}\{2011 \\
2014\}\end{array}$ & 1.2 & 1.16 & $\begin{array}{c}\mathrm{R}(\mathrm{A})>\mathrm{R}(\mathrm{B}) \\
0.95 \triangleleft R(A) / R(B) \mid<1.05\end{array}$ & $\begin{array}{l}\text { symmetric } \\
\text { mutualism }\end{array}$ & $\begin{array}{l}\text { The difference in the } \\
\text { dependence on the } \\
\text { collaboration } \\
\text { between the two is } \\
\text { very small }\end{array}$ \\
\hline
\end{tabular}

Figure 2 shows the histogram of the symbiosis degree of collaborative productivity between mentor and mentee; the $x$-axis represents the time in years, and the $y$-axis represents the symbiosis degree of productivity indicator based on the analysis of the symbiosis degree of collaborative productivity. In $1994-1995, R(A)>R(B)>0$, and that which reveals that there is an asymmetric mutualistic symbiosis relationship between the two academicians and that there is a large difference in terms of dependence on the collaboration between them. The mentee relied on the collaboration in the initial academic stage; collaborative papers contributed more to Li Shushen's scientific productivity. In 1996-2000, $R(B)>R(A)>0$, which indicates that the two subjects were both more dependent on the collaborative relationship; their relationship transformed to an asymmetric mutualistic symbiotic relationship. Although there are some differences between the two academicians in terms of the extent of their dependence on the collaboration, the difference is not too large, and their academic collaboration proceeded harmoniously. In 2001-2005, $R(A)>0>R(B)$, which indicates that the collaborative relationship between the two academicians in this period was beneficial to the scientific research output of Li Shushen; however, there was no advantage for the scientific research output of Xia Jianbai. After the mentor was appointed as an academician, the mentee became more dependent on collaboration with the mentor. Therefore, the symbiotic relationship between the two academicians at this time is of the form of commensalism. In 2006-2010, $R(B)>R(A)>0$, which indicates that there was a certain dependence on the collaborative relationship between the two academicians at this time. There is a difference in the dependence on the collaboration between the two, but this is not large, and the degree of dependence is small; the relationship between the two is an Page $\mid 68$ 
asymmetric mutualistic symbiotic relationship. In 2011-2014, $R(B)>R(A)>0$, which indicates that Xia Jianbai had a stronger dependence on the collaboration. There is a big difference between the two subjects in terms of the degree of dependence on the collaboration; the mentor had a strong dependence on the collaboration with the mentee in the later period of his academic career, and the relationship between the two is an asymmetric mutualistic symbiotic relationship. Yet, when considering the symbiosis degree of collaborative influence (measured by citation frequencies), one will find a different pattern (see in Figure 3).

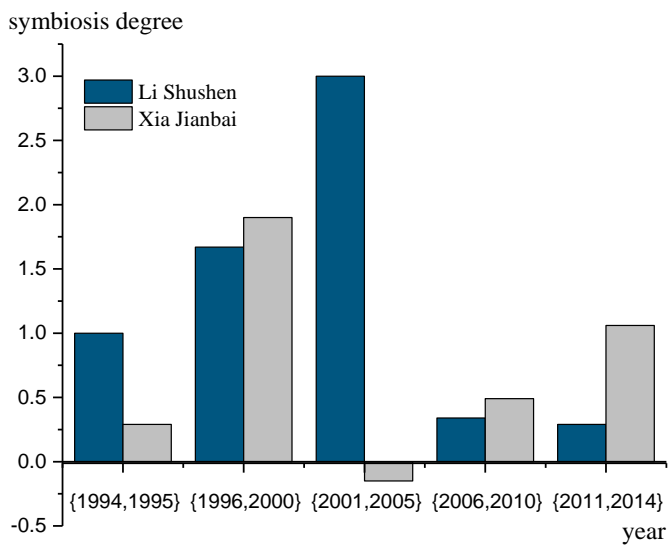

Figure 2. Symbiosis Degree of Collaborative Productivity between Mentor and Mentee

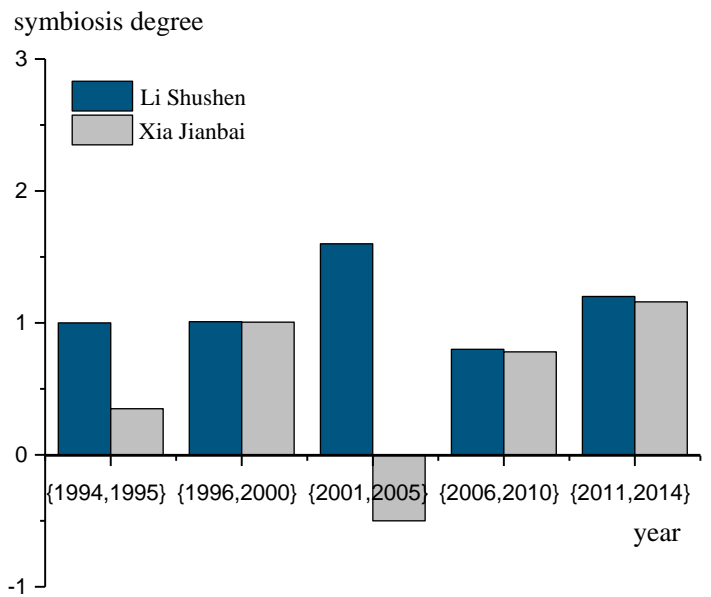

Figure 3. Symbiosis Degree of Collaborative Influence between Mentor and Mentee

From the above-mentioned example of a collaborative symbiosis between a mentor and mentee, we can see that the change in the number of collaborative papers affected the scientific research productivity of the two academicians. The young academician was more likely to benefit in 2001-2005, whereas in the other periods, the relationship between them was an asymmetric mutualistic symbiotic relationship. When they were in the initial stage of their collaboration, the young academician was more likely to benefit. However, with time, in the later stage of their collaboration, the old academician relied on the young academician 
to some extent. The number and the citation frequency of their collaborative papers have different impacts on the total number and the total citation frequency of the research papers of the two academicians; the symbiotic relationship between the two academicians appears to have varied in degree over time.

\section{CONCLUSIONS}

In this paper we have introduced a novel metric for analyzing the symbiotic relationship between subjects and demonstrate its potential for producing new insight into the nature of research collaboration. In our demonstration of the metric we found that: (a) the researchers in question did demonstrate some aspects of the "old leads young model," i.e., the young researchers draw support from the mentors' integrated resources to improve their productivity of scientific research, while developing their own networks and abilities, but also (b) A distinct evolution of the nature of the relationship over time between the mentor and mentee has obvious characteristics of evolution with time; at the beginning of their collaboration, the mentee relied more on the collaboration, whereas later on, the mentor's reliance on the collaboration becomes greater than the mentee's.

This study can serve as a reference for exploring the structure and characteristics of the symbiosis network of scientific collaboration and optimizing the selection of scientific partners in the process of scientific research, whether in mentor-mentee relationship, interdisciplinary collaboration, or other relationships.

While ultimately this is intended as a proof of concept for the method, the test case does have limitations. Firstly, this paper only chose Xia Jianbai and Li Shushen as an example of the symbiotic relationship between mentors and mentees. In later studies, we will continue to expand the number of research samples and the research field to make the research results more general. Secondly, we will study the interdisciplinary or multidisciplinary collaboration between scholars contrasting with symbiotic collaboration research. Thirdly, this paper only explores the changes of the symbiotic relationship between mentor and mentee before and after they elected as academicians respectively.

In future research, we will consider matching the symbiotic relationship types with career stages, for instance, the PhD period, the Postdoc period, the first years at Assistant Prof. level etc. (Barbuto, Story, Fritz, and Schinstock 2011; Bu et al. 2018c, 2018d). It could also be applied to analyzing the impact of interdisciplinary collaboration by determining the degree to which each collaborator benefits. Additionally, we believe that the model of symbiotic relationship between two collaborative subjects can be extended to the study of the symbiotic relationship of the ternary structure, further expanding the research objects and scope of the study and providing more data on symbiotic relationships in scientific collaboration.

Because this article shows a pilot study, we intend to conduct a regular study of the regularity of the mentor-mentee symbiosis model in the future. Future work is more about emphasizing methods, looking for quantitative research indicators, and whether further promotion to other areas requires further large sample empirical research. 


\section{ACKNOWLEDGMENTS}

This research received the financial support from National Natural Science Foundation of China: Study on the Structure and Evolution of the Scientific Collaboration Network of Academicians from the Perspective of Symbiosis: A Case Study of Academicians of CAS and NAS under grant number 71603015 . Also supported by the Natural Science Foundation of Beijing, China (Grant No. 9182001). Our gratitude also goes to the anonymous reviewers for their valuable comments.

\section{REFERENCES}

Albino V, Fraccascia L, and Giannoccaro I. 2015. Measuring complexity of industrial symbiosis networks. 10th International Forum on Knowledge Asset Dynamics, Bari, Italy; 277-292.

Barbuto, J.E., Jr., Story, J.S., Fritz, S.M., and Schinstock, J.L. 2011. Full range advising: Transforming the advisor-advisee experience. Journal of College Student Development, Vol. 52, no. 6: 656-670.

Borgatti, S.P., Everett, M.G., and Freeman, L.C. 2002. Ucinet for windows: Software for social network analysis. Harvard, MA: Analytic Technologies.

Bu Y, Ding Y, Liang X, and Murray D.S. 2018a. Understanding persistent scientific collaboration. Journal of the Association for Information Science and Technology, Vol. 69, no. 3: 438-448.

Bu Y, Murray D.S, Ding Y, Huang Y, and Zhao Y. 2018b. Measuring the stability of scientific collaboration. Scientometrics, Vol. 114, no. 2: 463-479.

Bu Y, Ding Y, Xu J, Liang X, Gao G, and Zhao Y. 2018c. Understanding success through the diversity of collaborators and the milestone of career. Journal of the Association for Information Science and Technology, Vol. 69, no. 1: 87-97.

Bu Y, Murray D.S, Xu J, Ding Y, Ai P, Shen J and Yang F. 2018d. Analyzing scientific collaboration with "giants" based on the milestones of career. In Proceedings of the 81st Annual Meeting of the Association for Information Science and Technology, Vancouver, British Columbia, Canada; 29-38.

Cavusoglu A. and Turker I. 2014. Patterns of collaboration in four scientific disciplines of the Turkish collaboration network. Physica A-Statistical Mechanics and its Applications, Vol. 413, 220-229.

Chen Y.W, Borner K. and Fang S. 2013. Evolving collaboration networks in Scientometrics in 1978-2010: a micro-macro analysis. Scientometrics, Vol. 95, no. 3: 1051-1070.

Chinchilla-Rodríguez Z, Bu Y, Robinson-Garcia N, Costas R and Sugimoto C R. 2018. Travel bans and scientific mobility: utility of asymmetry and affinity indexes to inform science policy. Scientometrics, Vol. 116, 569-590.

Clarivate Analytics. 2017. Derwent Data Analyzer. Available at: https://clarivate.com/products/derwent-data-analyzer/.

Ding, Y. 2011. Scientific collaboration and endorsement: Network analysis of coauthorship and citation networks. Journal of Informetrics, Vol. 5, no. 1: 187-203.

Domenech T and Davies M. 2011. Structure and morphology of industrial symbiosis networks: The case of Kalundborg. 4th and 5th UK Social Networks Conferences, London, England; 10.

Douglas, A.E. 1994. Symbiotic interactions. Oxford: Oxford University Press.

Egghe L and Rousseau R. 2003. A measure for the cohesion of weighted networks. Journal of the American Society for Information Science and Technology, Vol. 54, no. 3: 193-202. 
Feng, F., Xiao, X. and Zhang, L. 2013. Study on the classification and network construction of symbiosis in industry-university-research cooperation: based on principle of quality parameter compatibility depicted by extended logistic model. Science of Science and Management of Science and Technology, Vol. 34, no. 2: 3-11. (in Chinese)

Glänzel, W. 2002. Coauthorship patterns and trends in the sciences (1980-1998): A bibliometric study with implications for database indexing and search strategies. Library Trends, Vol. 50, no. 3: 461-473.

Heinrich R and Rapoport T.A. 1974. A linear steady-state treatment of enzymatic chains: General properties, control and effector strength. European Journal of Biochemistry, Vol. 42, no. 1: 89-95.

Hou, J., Zhang, S., and Pan, L. 2013. Detecting research fronts of international scientific collaboration. Science and Technology Management Research, no. 22: 32-37. (in Chinese)

Huerta-Barrientos A, Elizondo-Cortes M. and de la Mota I.F. 2014. Analysis of scientific collaboration patterns in the co-authorship network of Simulation-Optimization of supply chains. Simulation Modelling Practice and Theory, Vol. 46, no. 4: 135-148.

Kacser H. and Burns J. 1973. The control of flux. Symposia of the Society for Experimental Biology, Vol. 27: 65-104.

Kretschmer H., and Aguillo I. 2004. Visibility of collaboration on the Web. Scientometrics, Vol. 61, no. 3: 405-426.

Liu, J., Zheng, X., Wang, F., and Feng, X. 2015. Analysis on the age distribution of scientific elites' scientific productivity and influence: a case study of academicians of Chinese Academy of Science. Journal of Intelligence, Vol. 33, no. 11: 30-36. (in Chinese)

Milojević, S. 2010. Modes of collaboration in modern science: Beyond power laws and preferential attachment. Journal of the American Society for Information Science and Technology, Vol. 61, no. 7: 1410-1423.

Newman, M.E.J. 2001a. The structure of scientific collaboration networks. Proceedings of the National Academy of Sciences of the United States of America, Vol. 98, no. 2: 404-409.

Newman, M.E.J. 2001b. Scientific collaboration networks I. Network construction and fundamental results. Physical Review E, Vol. 64, no. 1: 016131(1-8).

Otte E., and Rousseau R. 2002. Social network analysis: a powerful strategy, also for the information sciences. Journal of Information Science, Vol. 28, no. 6: 443-455.

Qiu, J., and Yu, H. 2014. Analysis of scientific collaboration age structure of China information science. Information Science, no. 8: 3-8. (in Chinese).

Shi, W., Shen, J., and Wan, Y. 2013. A research on cooperation mechanism of inter-provincial sports scientific research in China from perspective of symbiosis theory. Journal of Shanghai Normal University (Natural Sciences), Vol. 42, no. 1: 55-64. (in Chinese)

Smith, A. 2011. Wikipedia and institutional repositories: an academic symbiosis? Proceedings of the International Conference on Scientometrics and Informetrics, Durban, South Africa; 794-800.

Wang, L., Thijs, B., and Glanzel, W. 2015. Characteristics of international collaboration in sport sciences publications and its influence on citation impact. Scientometrics, Vol. 105, no. 2: 843-862.

Wang, Z., and Yin, J. 2005. Research on operation pattern of industrial symbiosis network in eco-industry park. China Soft Science, no. 2: 80-85. (in Chinese)

Wang, Z., Tan, Q., and Xu, X. 2006. The evolution model and empirical studies of enterprises cluster symbiosis. Chinese Journal of Management Science, Vol. 14, no. 2: 141-148. (in Chinese)

Wang, Z., and Zhu, J. 2014. Homophily versus preferential attachment: Evolutionary mechanisms of scientific collaboration networks. International Journal of Modern Physics C, Vol. 25, no. 5: 323-337.

Page $\mid 72$ 
Williams, L. 2013. Modeling symbiosis by a Lotka-Volterra-type system of differential equations. Master's Thesis. Graduate School of the Texas Woman's University, ProQuest Dissertations Publishing (1523998).

Yuan, C. 1998. Symbiosis theory and its application to small economy (I). Reform, no. 2: 101105. (in Chinese)

Zhang, C., Bu, Y., Ding, Y., and Xu, J. 2018. Understanding scientific collaboration: Homophily, transitivity, and preferential attachment. Journal of the Association for Information Science and Technology, Vol. 69, no. 1: 72-86. 